\title{
The influence on the spin vectors of asteroids from the Yarkovsky effect
}

\author{
E. Skoglöv^ \\ Astronomiska Observatoriet, Box 515, 75120 Uppsala, Sweden
}

Received 12 April 2002 / Accepted 25 June 2002

\begin{abstract}
The spin vector evolution of a solar system object is connected to the orbital evolution of the body. The orbital and spin elements of the asteroids are affected by gravitational as well as thermal forces. When the spin rate, shape and orbital evolution of an object are known, the secular spin vector evolution may be determined. In this study, the orbital and spin vector evolutions of a number of artificial objects are numerically integrated, with special consideration taken to the seasonal and diurnal variants of the Yarkovsky force. The thermal force known as the Yarkovsky effect may perturb the orbital elements of an asteroid and thereby also affect the direction of the spin vector. Concentrating on spherical bodies, the spin axis evolution of bare and regolith covered main belt objects with radii larger than $50 \mathrm{~m}$ is examined, especially considering the seasonal and diurnal Yarkovsky forces. The combined effects on the spin vector evolution from these forces and induced periodical perturbations on the orbital elements are also studied. It is found that the effects on the spin vector evolution from the Yarkovsky force always are small or negligible for kilometer sized objects over time periods of $\sim 100 \mathrm{Myr}$ or more. The effects are doubled when the object radius is halved. During a given time period, the influences from the seasonal Yarkovsky force on the spin vector evolution of bare basaltic bodies are about 2-3 times as large as those on iron-rich ones. However, since the expected time, $t_{\text {rot }}$, before the spin axis direction is changed by a collisional event is about half as long for a bare basaltic object as for an equal sized iron-rich one, the seasonal Yarkovsky force may be of approximately the same importance for both classes of objects. The effects on regolith covered objects from this force are considerably smaller. On the other hand, the influences from the diurnal Yarkovsky effect are much stronger on regolith covered objects than on bare ones. The effect on the spin vector evolution from both variants of the Yarkovsky force seems to be small for all the objects studied unless very long time periods are considered. The size of $t_{\text {rot }}$ is dependent on several factors, but the influences from the Yarkovsky force on the spin axis evolution over the present age of the solar system are always negligible when the object radius is larger than $\sim 10 \mathrm{~km}$. Note that the effects studied in this paper should not be confused with the Yarkovsky-O'Keefe-Radzievskii-Paddack (YORP) effect.
\end{abstract}

Key words. minor planets, asteroids - celestial mechanics

\section{Introduction}

The diurnal variant of the "Yarkovsky force" was discovered by Ivan Yarkovsky a century ago but the first modern study of the effect was performed by Öpik (1951). Essentially, the Yarkovsky force is a recoil force due to thermally reemitted radiation from object regions of different temperatures. For the normal asteroid, the effect has a seasonal part related to the revolution around the Sun (e.g. Rubincam 1995, 1998) and a diurnal part related to the rotation of the object (e.g. Vokrouhlický 1998a, 1998b). Which variant of the force will be dominating for a particular object depends on the physical, rotational and thermal properties of the body (e.g. Farinella et al. 1998). The effects on the orbital evolution from both the diurnal and the seasonal Yarkovsky forces seem to be most important for objects with radii of approximately $(0.1-100) \mathrm{m}$.

\footnotetext{
* e-mail: Erik.Skoglov@astro.uu.se
}

Comparatively few objects in this size range are known observationally. However, a number of near-Earth objects with diameters of (10-100) $\mathrm{m}$ have been observed (e.g. Rabinowitz et al. 1993). Smaller objects are less affected by the Yarkovsky effect since they are more isothermal. Larger objects are too massive, with more mass per area, to be subjected to strong Yarkovsky accelerations.

Both force variants may be important for the transport of material from the asteroid main belt to the inner solar system. The Yarkovsky effect may be important in a slower phase, $\sim(10-100)$ Myr, transporting a body into a secular or mean motion resonance (e.g. Vokrouhlický \& Farinella 1998a; Bottke et al. 2000). The object may then fast evolve a planet crossing orbit.

One thermal effect, the Yarkovsky-O'Keefe-RadzievskiiPaddack (YORP) effect, may be important in changing both the spin rate and spin axis direction of certain asymmetrically 
shaped asteroids. Spherical and spheroidal bodies or triaxial ellipsoids will not be affected by the YORP effect (Rubincam 2000).

The aim of this study is to investigate the role of another effect related to the Yarkovsky force on the spin axis evolution of asteroids. The perturbation of the orbital evolution due to the Yarkovsky effect will also affect the spin axis evolution of the object. The orbital and spin elements of the asteroids are affected by gravitational and thermal forces. The Sun exerts a gravitationally related torque on all objects in the solar system, causing a precession motion of their spin vectors. The major planets are also affecting the orbital and spin vector evolutions of the asteroids by gravitationally related perturbations. When the spin rate, shape and orbital evolution of an object are known, the secular spin vector evolution may be determined, using the parameters $X=(\cos \eta)$ and $\psi$ of the spin vector direction. The obliquity parameter $\eta$ is defined as the tilt of the spin axis from the normal to the orbital plane and $\psi$ is the precession angle in longitude. In this study, the orbital and spin vector evolutions of a number of artificial objects are numerically integrated, with special consideration taken to the seasonal and diurnal variants of the Yarkovsky force.

From the standpoint of Yarkovsky force influences, asteroids can be considered to be "small" or "large". For a "large" object, the thermal penetration depth is small as compared to the object radius. For a smaller object however, one side will be thermally communicating with the other and the object will be more isothermal. The thermal penetration depth is depending on the thermal properties of the object, but an asteroid with a radius larger than $\sim 20 \mathrm{~m}$ can be considered large when considering both variants of the Yarkovsky force regardless of the material. The rotational properties of real solar system bodies that can be considered "small" in this respect are almost totally unknown. The objects with known spin properties are typically kilometer sized and larger. No thermally "small" objects will be considered in this study, note also that for such objects the Poynting-Robertson force is becoming increasingly more important with diminishing radius. Also, since the objects are assumed to be spherical, the YORP effect may be ignored.

\section{The effects from the Yarkovsky force on the orbital evolution}

\subsection{The diurnal Yarkovsky force}

The reasoning when examining the diurnal and seasonal effects is similar (e.g. Vokrouhlický 1998a, 1998b; Rubincam 1995, 1998). The forces are somewhat different though, e.g. will the seasonal effect always decrease the semimajor axis of the orbit, an effect known as thermal drag, at least when the orbital eccentricity is not very high (Spitale \& Greenberg 2001), while the diurnal effect will cause the orbit to expand for a prograde rotator and to shrink for an object with a retrograde rotation. The influences on the other orbital elements are in a similar way dependent on the orbital and spin parameters and on which Yarkovsky force variant is considered (see also e.g. Bottke et al. 2000; Spitale \& Greenberg 2002).
In this study, the concentration will be on spherical bodies with circular orbits. Also, the spin periods are supposed to be short as compared to the orbital periods.

The method used by Vokrouhlický (1998a) will be followed in order to examine the diurnal effects on the orbital elements.

In a solid medium, the heat conduction equation can be described by:

$\rho C(\partial T / \partial t)=K \nabla^{2} T$

where $\rho$ is the material density, $C$ is the specific heat, $K$ is the thermal conductivity and $\nabla^{2}$ is the Laplace operator

This gives the temperature $T$ throughout the medium at any time $t$.

A boundary condition on the surface of the object is also needed:

$E \sigma T^{4}+K(\boldsymbol{n} \cdot(\partial T / \partial \mathbf{r}))=A_{\mathrm{abs}} \epsilon$

where $E$ is the emissivity, $\sigma$ is the Stefan-Boltzmann constant, $\boldsymbol{n}$ is the unit vector normal to the body's surface, $(\boldsymbol{n} \cdot(\partial T / \partial \boldsymbol{r}))$ describes the temperature variance inside the body, $A_{\mathrm{abs}}$ is the absorption coefficient and $\epsilon$ is the external radiation flux.

The first left-hand term describes the energy thermally reradiated by the body while the second term is the energy conducted to deeper body layers. The right-hand side gives the energy entering the unit surface area of the body per unit time.

Generally, the exact appearance of the thermal force will depend on several factors. Among these are the shape of the body and whether or not the body is dynamically relaxed, i.e. in stable rotation around the shortest semi-axis of the object. In this study, a dynamically relaxed spherical body having a radius $R$ and an angular velocity of the rotation, $\omega$, is assumed.

If $\epsilon_{*}$ is the solar radiation flux at the position of the object, an auxiliary temperature $T_{*}$ can be defined:

$T_{*}=\left[A_{\mathrm{abs}} \epsilon_{*} /(E \sigma)\right]^{1 / 4}$.

Now, the thermal parameter, $\Theta$, and the dimensionless scaled radius, $R^{\prime}$, are:

$\Theta=\frac{(\rho C K \omega)^{1 / 2}}{E \sigma T_{*}{ }^{3}}$

$R^{\prime}=R / l_{\mathrm{S}}$

where $l_{\mathrm{S}}$ is the thermal length:

$l_{\mathrm{S}}=[K /(\rho C \omega)]^{1 / 2}$.

Now define:

$\lambda \equiv \Theta /\left(2^{1 / 2} R^{\prime}\right)$.

Use the auxiliary real functions $A(x), B(x), C(x), D(x), E(x)$ and the phase $\delta(x)$ :

$$
\begin{aligned}
A(x)= & -(x+2)-\mathrm{e}^{x}[(x-2) \cos x-x \sin x] \\
B(x)= & -x-\mathrm{e}^{x}[(x-2) \sin x+x \cos x] \\
C(x)= & A(x)+[\lambda /(1+\lambda)]\{3(x+2) \\
& \left.+\mathrm{e}^{x}[3(x-2) \cos x+x(x-3) \sin x]\right\}
\end{aligned}
$$


$D(x)=B(x)+[\lambda /(1+\lambda)]\{x(x+3)$

$$
\left.-\mathrm{e}^{x}[x(x-3) \cos x-3(x-2) \sin x]\right\}
$$

$E(x) \mathrm{e}^{i \delta(x)}=\frac{A(x)+i B(x)}{C(x)+i D(x)}$.

Let $E_{R^{\prime}}=E\left(2^{1 / 2} R^{\prime}\right)$ and $\delta_{R^{\prime}}=\delta\left(2^{1 / 2} R^{\prime}\right)$.

Now the thermal force per unit of body mass, $f$, can be expressed. If an non-rotating $X, Y, Z$ reference system is introduced (Vokrouhlický 1998a), with the $Z$-axis aligned with the spin axis of the object and the solar position permanently in the $X Z$-plane, the three components of $f$ are (assuming isotropic thermal emission):

$f_{X}+i f_{Y}=-\frac{4 A_{\mathrm{abs}}}{9} \Phi \frac{\sin \theta_{0}}{1+\lambda} E_{R^{\prime}} \mathrm{e}^{-i \delta_{R^{\prime}}}$

$f_{Z}=-\frac{4 A_{\mathrm{abs}}}{9} \Phi \frac{\cos \theta_{0}}{1+\lambda}$

where $\theta_{0}$ is the solar colatitude and

$\Phi \equiv\left(\pi R^{2} \epsilon_{*}\right) /(m c)$

where $m$ is the object mass and $c$ is the speed of light

Obviously, $\boldsymbol{f}$ will decrease with increasing body mass. The importance of the effect will also be diminished when the size of the body is increased.

Now, the secular perturbations on $a$, the semi-major axis of the orbit, and $I$, the orbital inclination, can be calculated:

$(\mathrm{d} a / \mathrm{d} t)=-\frac{8 A_{\mathrm{abs}}}{9} \frac{\Phi}{n} \frac{E_{R^{\prime}} \sin \delta_{R^{\prime}}}{1+\lambda}(\boldsymbol{s} \cdot \boldsymbol{N})$

$(\mathrm{d} I / \mathrm{d} t)=\frac{2 A_{\mathrm{abs}}}{9} \frac{\Phi}{n a}$

$\times \frac{(\boldsymbol{s} \cdot \boldsymbol{P})\left(1-E_{R^{\prime}} \cos \delta_{R^{\prime}}\right)(\boldsymbol{s} \cdot \boldsymbol{N})+(\boldsymbol{s} \cdot \boldsymbol{Q})\left(E_{R^{\prime}} \sin \delta_{R^{\prime}}\right)}{1+\lambda}$

$(\boldsymbol{s} \cdot \boldsymbol{N})=\cos \eta=X$

$(\boldsymbol{s} \cdot \boldsymbol{P})=\sin \psi\left(1-X^{2}\right)^{1 / 2}$

$(\boldsymbol{s} \cdot \boldsymbol{Q})=\cos \psi\left(1-X^{2}\right)^{1 / 2}$

where $n$ is the object's orbital mean motion around the Sun, $n=\left(G M a^{-3}\right)^{1 / 2}, G$ is the gravitational constant, $M$ is the solar mass, $\boldsymbol{s}$ is the spin vector, $\boldsymbol{N}$ is the unit vector normal to the mean orbit, $\boldsymbol{P}$ is the position vector of the mean pericenter and $\boldsymbol{Q}=\boldsymbol{N} \times \boldsymbol{P}$. The spin parameter $\eta$ is the obliquity, i.e. the tilt of the spin axis from the normal to the orbital plane and $\psi$ is the precession angle in longitude. In Eqs. (16) and (17), a circular orbit is assumed. Also, the rotation frequency of the object around its spin axis is assumed to be much larger than the mean motion frequency of the object around the Sun. A more general solution can be found in Vokrouhlický (1999).

\subsection{The seasonal Yarkovsky force}

A more general model, treating the asteroids as spheres (e.g. Rubincam 1998), must be used for thermally "small" objects. However, since only thermally "large" objects are considered, a plane-parallel model can be used as a valid approximation for the bodies in this study. Thus, the method used by Rubincam (1995) as modified by Bottke et al. (2000) will be followed in order to examine the seasonal Yarkovsky effect on the orbital elements.

In a solid medium, using the plane-parallel model, the heat conduction equation can be described by:

$\rho C(\partial T / \partial t)=K\left(\partial^{2} T / \partial z^{2}\right)$

where $\rho$ is the material density, $C$ is the specific heat, $K$ is the thermal conductivity and $z$ is the depth below the surface (positive downward).

This gives the temperature $T$ throughout the medium at any time $t$.

The boundary condition on the surface of the object can now be expressed as:

$E \sigma T^{4}-K(\partial T / \partial z)_{z=0}=(1-A) \epsilon$

where $E$ is the emissivity, $\sigma$ is the Stefan-Boltzmann constant, $(\partial T / \partial z)$ describes the temperature variance inside the body, $A$ is the albedo and $\epsilon$ is the external radiation flux.

Still, a spherical body having a radius $R$ and an angular velocity of the rotation, $\omega$, is assumed.

$T$ can now be expressed as a sum of an average temperature, $T_{0}$, constant throughout the object and a term $\Delta T$, containing all time-like and space-like temperature variations.

Using a Taylor expansion, we have the relation:

$T^{4} \approx T_{0}^{4}+4 T_{0}^{3} \Delta T$.

Now, $T_{0}$ is found from:

$E \sigma T_{0}{ }^{4}=(1-A)\left(\epsilon_{\mathrm{E}} / 4\right)\left(a_{\mathrm{E}} / a\right)^{2}\left(1-e^{2}\right)^{-1 / 2}$

where $a$ and $e$ are the semi-major axis and eccentricity of the asteroid's orbit, $a_{\mathrm{E}}$ is the semi-major axis of the orbit of the Earth and $\epsilon_{\mathrm{E}}$ is the solar constant when $a=a_{\mathrm{E}},\left(\epsilon_{\mathrm{E}}=\right.$ $1378 \mathrm{~W} \mathrm{~m}^{-2}$ ).

Finding $\Delta T$ is a more complicated process. For the details of this and the general time evolution of the orbital elements given by Lagrange's planetary equations, see e.g. Rubincam (1995).

If the longitude of the ascending node and the argument of perihelion of the orbit is circulating uniformly, and circular orbits are assumed, the secular perturbations from the seasonal Yarkovsky force on the semi-major axis, $a$, and inclination, $I$, of the asteroid's orbit can be calculated after averaging over the orbital period (Rubincam 1995; Bottke et al. 2000):

$$
\begin{aligned}
& (\mathrm{d} a / \mathrm{d} t)=-\frac{(1-A) \epsilon_{\mathrm{E}}}{3 c \rho R n} \frac{a_{\mathrm{E}}^{2}}{a^{2}} \frac{\Phi_{1}}{1+2 \Phi_{1}+2 \Phi_{1}{ }^{2}}\left(1-X^{2}\right) \\
& (\mathrm{d} I / \mathrm{d} t)=\frac{(1-A) \epsilon_{\mathrm{E}}}{24 c \rho R n} \frac{a_{\mathrm{E}}^{2}}{a^{3}} \sin (2 I) \frac{\Phi_{1}}{1+2 \Phi_{1}+2 \Phi_{1}{ }^{2}}\left(1-3 X^{2}\right)
\end{aligned}
$$


where $n$ is the object's mean motion around the Sun, $R$ and $\rho$ are the object's radius and material density and $c$ is the speed of light

The thermal function $\Phi_{1}$ is defined as:

$\Phi_{1}=\frac{(K \rho n C)^{1 / 2}}{(32)^{1 / 2} E \sigma T_{0}^{3}}$

$X=\cos \eta$, where $\eta$ is the obliquity.

A larger body radius will decrease the effect on the orbital elements. The seasonal Yarkovsky effect will thus be negligible when the body radius is very large.

As noted by Rubincam (1995), a linearized solution will slightly overestimate the influences from the seasonal Yarkovsky force. According to the nonlinearized model used by Vokrouhlický \& Farinella (1998b), this overestimation is $\sim 15 \%$ for thermally large objects.

Other representations of the semi-major axis change due to the seasonal Yarkovsky force can be seen e.g. in Farinella et al. (1998) and in Vokrouhlický (1999).

\section{The connection between the orbital and spin vector evolutions}

The evolution of the spin axis of a solar system object is connected to the orbital evolution. Several equivalent representations of the spin vector evolution equations are possible. The spin vector evolution of the major planets was studied by Laskar \& Robutel (1993) and Laskar et al. (1993). As for the derivation of the equations of spin vector evolution, see e.g. these studies and the references given there. In Skoglöv et al. (1996) and in Skoglöv (1997), the equations for the spin vector evolution were adapted for the asteroids.

The orbital and spin elements of the asteroids are affected by gravitational as well as thermal forces. The Sun exerts a gravitationally related torque, $\boldsymbol{L}$, on all objects in the solar system. This torque is also causing a precession motion of the spin vectors of the objects. Limited to first order in $R / r$ (the equatorial radius of the object divided by the distance to the Sun), the torque can be approximated as:

$\boldsymbol{L}=\frac{3 G M}{r^{3}} \hat{\boldsymbol{r}} \times J \cdot \hat{\boldsymbol{r}}$

where $\hat{\boldsymbol{r}}$ is the unit vector in the direction of the Sun, $G$ is the gravitational constant, $M$ is the solar mass and $J$ is the inertia tensor.

The orbital evolution of a real asteroid is also subjected to gravitational perturbations from the major planets. Knowing the orbital evolution, it is possible to integrate the secular spin vector evolution numerically using the parameters $X$ and $\psi$ of the spin vector direction. If the obliquity parameter $\eta$ is defined as the tilt of the spin axis from the normal to the orbital plane and $\psi$ is the precession angle in longitude, then $X=\cos \eta$. The basic equations of precession used in the spin vector integrations are:

$$
\begin{aligned}
\mathcal{H}(X, \psi, t)= & (\alpha / 2)\left(1-e(t)^{2}\right)^{-3 / 2} X^{2}+\left(1-X^{2}\right)^{1 / 2}(A(t) \sin \psi \\
& +B(t) \cos \psi)-2 C(t) X
\end{aligned}
$$

with

$$
\begin{aligned}
A(t)= & 2(\dot{q}+p(q \dot{p}-p \dot{q})) /\left(1-p^{2}-q^{2}\right)^{1 / 2}=(\mathrm{d} I / \mathrm{d} t) \cos \Omega \\
& -(\mathrm{d} \Omega / \mathrm{d} t) \sin I \sin \Omega
\end{aligned}
$$

$$
\begin{aligned}
B(t)= & 2(\dot{p}-q(q \dot{p}-p \dot{q})) /\left(1-p^{2}-q^{2}\right)^{1 / 2}=(\mathrm{d} I / \mathrm{d} t) \sin \Omega \\
& +(\mathrm{d} \Omega / \mathrm{d} t) \sin I \cos \Omega
\end{aligned}
$$

$C(t)=(q \dot{p}-p \dot{q})=(\mathrm{d} \Omega / \mathrm{d} t) \sin ^{2}(I / 2)$

$p=\sin (I / 2) \sin (\Omega)$

$q=\sin (I / 2) \cos (\Omega)$

$\dot{p}=(\partial p / \partial t)$

$\dot{q}=(\partial q / \partial t)$

$$
\begin{aligned}
(\mathrm{d} \psi / \mathrm{d} t)= & \alpha\left(1-e(t)^{2}\right)^{-3 / 2} X-X\left(1-X^{2}\right)^{-1 / 2}(A(t) \sin \psi \\
& +B(t) \cos \psi)-2 C(t)
\end{aligned}
$$

$(\mathrm{d} X / \mathrm{d} t)=-\left(1-X^{2}\right)^{1 / 2}(A(t) \cos \psi-B(t) \sin \psi)$

where $\mathcal{H}$ is the Hamiltonian associated with the spin angular momentum of the object, $t$ is the time, $I$ is the orbital inclination, $e$ is the orbital eccentricity and $\Omega$ is the longitude of the ascending node, $(\mathrm{d} \psi / \mathrm{d} t)=(\partial \mathcal{H} / \partial X),(\mathrm{d} X / \mathrm{d} t)=-(\partial \mathcal{H} / \partial \psi)$.

The term containing $C(t)$ is usually small and may often be omitted when examining the spin vector evolution of asteroids, in particular when comparing with the uncertainties in the spin parameter $\alpha$. However, since small model related differences are examined, it will always be included in the spin vector integrations in this study.

The precession parameter $\alpha$ depends on several object properties (e.g. Laskar \& Robutel 1993; Laskar et al. 1993; Skoglöv et al. 1996):

$\alpha=\frac{3 G M \gamma}{2 \omega a^{3}}=\frac{3 \pi \gamma P_{\text {spin }}}{P_{\text {orb }}^{2}}$

where $G$ is the gravitational constant, $M$ is the solar mass, $\omega$ is the angular spin velocity, $a$ is the semi-major axis of the orbit, $\gamma$ is the dynamical ellipticity, $P_{\text {spin }}$ is the spin period and $P_{\text {orb }}$ is the orbital period. The precession parameter may be variable, e.g. if the semi-major axis $(a)$ changes considerably over time. This is the case for many inner solar system asteroids.

The dynamical ellipticity $(\gamma)$ depends on the shape and internal mass distribution of the considered object. Often, asteroids have been modelled as homogeneous ellipsoidal objects with semi-axes $a \geq b \geq c$, in stable rotation around the shortest $c$-axis. If the spin frequency is much higher than the precession frequency, the dynamical ellipticity can be defined as:

$\gamma=\frac{1}{2}-\frac{c^{2}}{a^{2}+b^{2}}$. 


\section{The spin vector evolution}

\subsection{Spin vector integration excluding the Yarkovsky force}

The normal rotational conditions of real asteroids with $R<$ $1 \mathrm{~km}$ are almost totally unknown. The objects in this study are assumed to be dynamically relaxed, their spin vectors are not subjected to changes that are fast as compared to the orbital period, e.g. wobbling and tumbling motions are not considered. It is not clear to what extent this is normal for real subkilometer sized bodies. The time scale for damping to a stable rotation around the principal axis depends on several object properties, including the spin rate, size, shape and material density (Burns \& Safronov 1973). Small, slowly rotating objects may have very long damping times (Harris 1994). The damping time may thus be very different for various objects. However, following a sudden spin change due to a collision or near passage, the typical relaxation time for subkilometer sized bodies may be in the order of $\sim(1-20)$ Myr.

The thermal parameters $\rho$, the material density, $C$, the specific heat and $K$, the thermal conductivity, depend on the body material. The values in this study are the same as in Farinella et al. (1998), but can also be found e.g. in Rubincam (1995) for basaltic and regolith-covered materials and in Burns et al. (1979) for iron-rich material.

basaltic material:

$K=2.65 \mathrm{Wm}^{-1} \mathrm{~K}^{-1}, \rho=3500 \mathrm{~kg} \mathrm{~m}^{-3}, C=680 \mathrm{~J} \mathrm{~kg}^{-1} \mathrm{~K}^{-1}$

iron-rich material:

$K=40 \mathrm{Wm}^{-1} \mathrm{~K}^{-1}, \rho=8000 \mathrm{~kg} \mathrm{~m}^{-3}, C=500 \mathrm{~J} \mathrm{~kg}^{-1} \mathrm{~K}^{-1}$

regolith-covered material:

$K=0.0015 \mathrm{Wm}^{-1} \mathrm{~K}^{-1}, \rho=1500 \mathrm{~kg} \mathrm{~m}^{-3}, C=680 \mathrm{~J} \mathrm{~kg}^{-1} \mathrm{~K}^{-1}$.

For a real regolith-covered object, it may be more realistic instead to use a bulk density of $\rho=3500 \mathrm{~kg} \mathrm{~m}^{-3}$, assuming a stony object covered by a regolith with the values of $C$ and $K$ seen above. Also, in the present study it is always assumed that in case of regolith-covered bodies the regolith is so deep that the longest period thermal pulses never sense the bottom of the regolith layer. A more rigorous treatment may be found in Vokrouhlický \& Broz (1999).

In addition, treating the objects as blackbodies, the emissivity $E=1$, the albedo $A=0$ and the absorption coefficient $A_{\mathrm{abs}}=1$.

For a perfect sphere, the three body axes are related as $a=b=c$. Thus, according to Eqs. (39) and (40), $\gamma=\alpha=0$ and the direct influences on the spin vector evolution from variations in $a$ and $e$ disappear. As can be seen in Skoglöv \& Erikson (2002), the variations and size of the orbital inclination, $I$, are the most important causes for the size of the regular obliquity changes of main belt objects (see also Fig. 1). Note that when the Yarkovsky effects are calculated, the orbital inclination evolution depends on the evolution of $a$ (Eqs. (17) and (26)). The variations in $a$ and $I$ used in this study also assume $e \equiv 0$.
The spin vector evolution depends on the orbital evolution. The evolution of the orbital elements of an object due to Yarkovsky forces is also dependent on the direction of the spin axis. Since the aim of this study is to examine the spin axis evolution due to the same forces, when the Yarkovsky effect is included the orbital and spin vector evolutions must be integrated simultanously, combining the orbital evolution due to the Yarkovsky force with the connection between the orbital and spin vector evolutions described in the earlier section. Using some suitable initial values, all spin vector evolutions were integrated numerically with a time step of $3.125 \mathrm{yr}$, a time step that was found necessary and sufficient.

The evolutions were integrated using an initial semi-major axis of the orbit of $3 \mathrm{AU}$, five different initial orbital inclinations, $0.0001^{\circ}, 2.5^{\circ}, 5^{\circ}, 10^{\circ}$ and $20^{\circ}$, and, when the Yarkovsky force was included, three different object radii, $50 \mathrm{~m}, 100 \mathrm{~m}$ and $1000 \mathrm{~m}$ with the sets of thermal parameters mentioned above. The situation for five different initial $X$-values, $X=0$, $X= \pm 0.5$ and $X= \pm 0.9$, was investigated. The initial $\psi=0$.

Due to gravitational and thermal forces, the orbits of the real solar system objects are variable. Initially, when the Yarkovsky force was excluded, all orbital elements except $\Omega$, the longitude of the ascending node, were kept constant.

In all integrations, $\Omega$ was assumed to be circulating, a full $360^{\circ}$ in 25000 years, rather than librating. For real main belt asteroids with $a$ in the range $[2.5,3.5] \mathrm{AU}$, this seems to be a reasonable assumption. When the other orbital elements are perturbed, by gravitational or Yarkovsky forces, $(\mathrm{d} \Omega / \mathrm{d} t)$ may also change. However, since the results were not affected significantly by any reasonable changes in $(\mathrm{d} \Omega / \mathrm{d} t)$ for the perturbed models, this will be ignored. The initial eccentricity, $e$, was assumed to be zero, i.e. the orbits were circular. Since the perturbations in the eccentricity due to the Yarkovsky effect are proportional to the eccentricity itself, i.e. $(\mathrm{d} e / \mathrm{d} t) \propto e$ (e.g. Rubincam 1995, 1998; Vokrouhlický 1998a), such an orbit will stay circular, $(\mathrm{d} e / \mathrm{d} t) \equiv 0$, also when Yarkovsky forces are included.

First, the Yarkovsky forces were ignored and the orbital inclination and semi-major axis stayed constant during the integration period, $\Omega$ was circulating. The obliquity variations are larger when the orbital inclination is higher (Fig. 1), something that also was noted by Skoglöv \& Erikson (2002).

\subsection{Spin vector integration including the seasonal Yarkovsky force}

The orbital and spin vector evolutions were integrated numerically, using the same initial values as before but now including the influences from the seasonal Yarkovsky force. The influences on $a$ and $I$ from other forces were ignored.

The differences in $X$ between the spin axis evolutions excluding and including the seasonal Yarkovsky force, $\Delta X_{\text {Yar,s }}$, can be seen in Fig. 2 for some typical objects. The result can be described as an oscillating function with the amplitude increasing with time and decreasing with a larger object radius. The frequency of the oscillation depends on the size of $(\mathrm{d} \Omega / \mathrm{d} t)$, 
so that faster changes in $\Omega$ decrease the period. The amplitude increase of $\Delta X_{\text {Yar,s }}$ is independent of the rate of $(\mathrm{d} \Omega / \mathrm{d} t)$.

The effects on the spin vector evolution from the seasonal Yarkovsky force are proportional to the orbital inclination perturbations for the different materials and were largest for the bare basaltic objects, about 2.6 times smaller for the iron-rich objects and $\sim 14$ times smaller for the regolith covered objects $\left(\rho=1500 \mathrm{~kg} \mathrm{~m}^{-3}\right)$. With a higher bulk density, $3500 \mathrm{~kg} \mathrm{~m}^{-3}$, the effects on the regolith-covered asteroids were $\sim 1.5$ times smaller than for the lower density.

The seasonal Yarkovsky force acting on the semi-major axis of the orbit is most effective when the spin axis of the body is in the orbital plane, due to large seasonal differences. When the axis is normal to the orbital plane, $X= \pm 1$, there is no acting thermal drag (Eq. (25)). However, the effects on the orbital inclination evolution are largest for $X= \pm 1$ (Eq. (26)), see also Rubincam (1995) and Bottke et al. (2000). Note that the influence on the orbital inclination changes sign when $X= \pm 3^{-1 / 2} \approx \pm 0.58$. The seasonal Yarkovsky force will increase the inclination when $X^{2}<3^{-1}$ and decrease it when $X^{2}>3^{-1}$. The sizes of $(\mathrm{d} a / \mathrm{d} t)$ and $(\mathrm{d} I / \mathrm{d} t)$ due to the seasonal Yarkovsky force depend on several factors, including some that are time variable. For a bare basaltic object with $I \approx 2.5^{\circ}$ and $R=50 \mathrm{~m}, a$ may decrease by typically $\left(10^{-4}-10^{-3}\right)$ AU over a period of 1 Myr. During the same time period, the typical changes in $I$ are approximately $\left(0^{\prime \prime}-3^{\prime \prime}\right)$.

For the objects in this study, the deviations due to the seasonal Yarkovsky force tend to increase with the inclination, regardless of initial $X$. For a given $X$-value, the effect on the orbital inclination is largest for $I=45^{\circ}$, (Eq. (26)). Also, the spin axis variations not related to the Yarkovsky effect are larger when the orbital inclination is higher, Fig. 1, and when initial $X$ is close to 0 (see also Skoglöv \& Erikson 2002). Thus, the influences from the Yarkovsky force will then be very variable over time for a given initial $X$. These effects may explain the larger $\Delta X_{\text {Yar,s }}$ found when the orbital inclination is higher.

The results for the 100 and 1000 meter radius objects are similar to those for the $50 \mathrm{~m}$ radius objects in that $\Delta X_{\text {Yar,s }}$ vary in an oscillating way. However, $\Delta X_{\text {Yar,s }}$ is halved when the object radius is doubled. This is expected, since the effects on $(\mathrm{d} a / \mathrm{d} t)$ and $(\mathrm{d} I / \mathrm{d} t)$ are halved in the same way when the radius is doubled and the other parameters are kept constant (see Eqs. (25) and (26)).

After $1 \mathrm{Myr}$, assuming a $50 \mathrm{~m}$ radius bare basaltic body, depending on initial $X$, the amplitude of $\Delta X_{\text {Yar,s }}$ is $\sim\left(10^{-11}-10^{-10}\right)$ for an initial $I=0.0001^{\circ},(0.4-3.6) \times 10^{-6}$ for an initial $I=2.5^{\circ}$ and increasing to $(0.8-1.8) \times 10^{-5}$ for an initial $I=20^{\circ}$. The sizes of the deviations are usually largest for initial $X=0$. In some cases, when initial $I=20^{\circ}$, they may be slightly larger for other initial $X$. A typical situation can be seen in Fig. 2 .

\subsection{Spin vector integration including the diurnal Yarkovsky force}

The spin vector evolution with the diurnal Yarkovsky force as perturbing force was integrated analogous to the earlier investigations. The differences between the spin axis evolutions
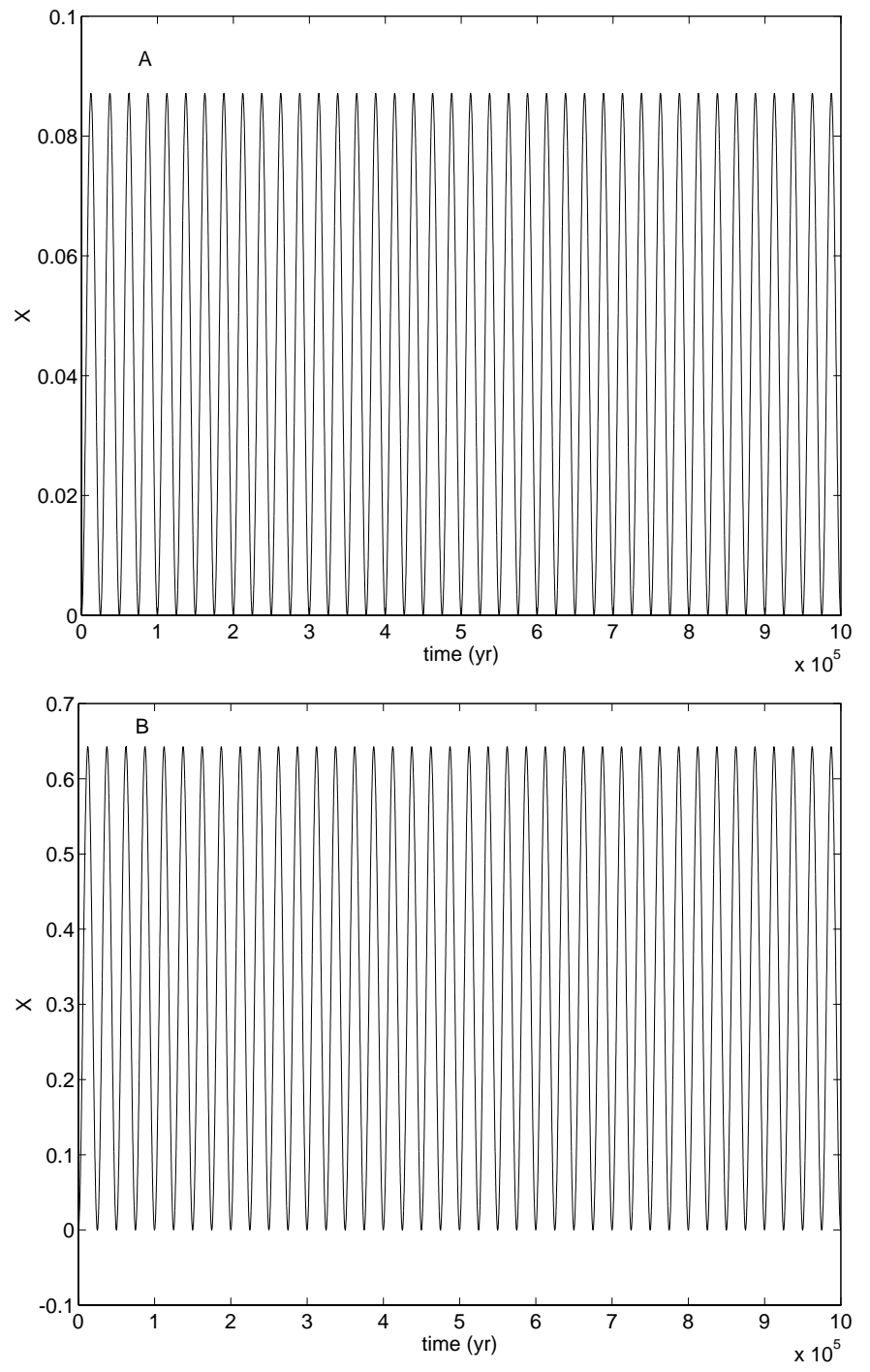

Fig. 1. The $X$-values ( $X=\cos \eta$; where $\eta$ is the obliquity) obtained for a time period of $1 \mathrm{Myr}$ for two hypothetical objects. Two constant orbital inclinations $(I)$ are used: A: $I=2.5^{\circ}$ and B: $I=20^{\circ}$. The semimajor axis of the orbit $(a)$ is constant, $a=3 \mathrm{AU}$. For both objects, the initial $X=0$. The longitude of the ascending node $(\Omega)$ is circulating a full $360^{\circ}$ in $25000 \mathrm{yr}$. The time-step of the spin-axis integration is $3.125 \mathrm{yr}$. The precession parameter $\alpha=0$. The initial precession angle in longitude $(\psi)$ is 0 . The tendency with increasing variations in $X$ with increasing inclination can be seen.

excluding and including the diurnal Yarkovsky force, $\Delta X_{Y a r, d}$, can be seen in Fig. 3 for some objects. As when the seasonal effects were examined, the result can be described as an oscillating function, usually with the amplitude increasing uniformly with time. As for the seasonal Yarkovsky force, the frequency of the oscillation depends on the size of $(\mathrm{d} \Omega / \mathrm{d} t)$, so that faster changes in $\Omega$ will decrease the period. The amplitude of the $\Delta X_{\text {Yar, }}{ }^{-O s c i l l a t i o n}$ is halved when the object radius is doubled provided the other factors, including the rotation rate, are kept constant and is independent of $(\mathrm{d} \Omega / \mathrm{d} t)$.

The amplitude of the $\Delta X_{\text {Yar, } \mathrm{d} \text {-oscillation is dependent on }}$ many factors, including the object radius, rotation rate, orbital inclination, material and the initial $X$ used. For a $50 \mathrm{~m}$ radius regolith-covered object with $\rho=1500 \mathrm{~kg} \mathrm{~m}^{-3}$, the amplitude 

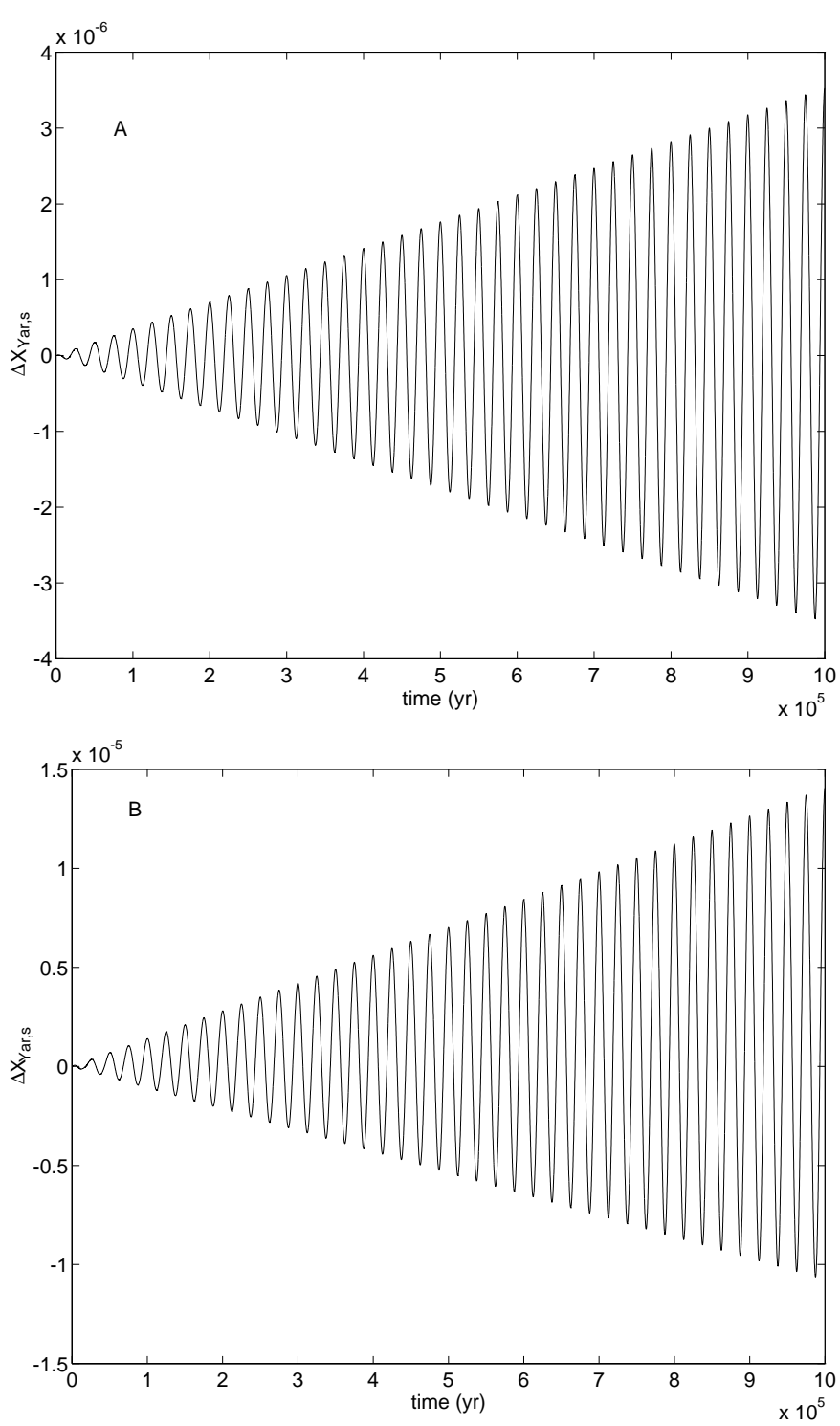

Fig. 2. The differences, $\Delta X_{\text {Yar,s }}$, between the spin vector evolutions excluding and including the seasonal Yarkovsky force $(X=\cos \eta$; where $\eta$ is the obliquity) obtained for a time period of $1 \mathrm{Myr}$ for two hypothetical bare basaltic objects. Two initial orbital inclinations $(I)$ are used: A: $I=2.5^{\circ}$ and B: $I=20^{\circ}$. For both objects, the initial $X=0$ and the initial semi-major axis of the orbit $(a)$ is $3 \mathrm{AU}$. The longitude of the ascending node $(\Omega)$ is circulating a full $360^{\circ}$ in $25000 \mathrm{yr}$. The radius of each object is $50 \mathrm{~m}$. The time-step of the spin-axis integration is $3.125 \mathrm{yr}$. The precession parameter $\alpha=0$. The initial precession angle in longitude $(\psi)$ is $0 . \Delta X_{\text {Yar, }}$ is oscillating with an amplitude that increases with time and orbital inclination and with a period depending on the size of $(\mathrm{d} \Omega / \mathrm{d} t)$.

of $\Delta X_{\text {Yar, }}$ is $\sim\left(5 \times 10^{-5}-4 \times 10^{-4}\right)$ after $1 \mathrm{Myr}$, depending on the initial inclination and the rotational parameters. Assuming equal sized objects with a rotational period of $0.5 \mathrm{~h}, \Delta X_{\text {Yar,d }}$ is about 3 times smaller for a regolith-covered object with a bulk density of $3500 \mathrm{~kg} \mathrm{~m}^{-3}, \sim 10^{2}$ times smaller for bare basaltic objects and $\sim 10^{3}$ times smaller for bare iron-rich asteroids.

The situation for several rotational periods between $0.5 \mathrm{~h}$ and $20 \mathrm{~h}$ was examined. It was found that this factor did affect $\Delta X_{\text {Yar,d }}$ to some extent. The smallest $\Delta X_{\text {Yar,d }}$ was generally obtained for a rotational period of $0.5 \mathrm{~h}$ and the largest for a period of $\sim(5-20) \mathrm{h}$. However, for the regolith-covered objects with $\rho=1500 \mathrm{~kg} \mathrm{~m}^{-3}$, where the importance of the diurnal Yarkovsky force is greatest, the increase in $\Delta X_{\text {Yar, }}$ was only about 1.4 times when the period was increased from $0.5 \mathrm{~h}$ to $5 \mathrm{~h}$.

For a fast rotator, an increase in rotation rate will decrease the temperature differences between day and night and the diurnal Yarkovsky effect will decrease in importance. Note however that for a very slow rotator an increase in rotation rate will increase the thermal lag angle while the effects on the temperature distribution will be negligible. In this case, the importance of the diurnal Yarkovsky effect may be increased.

The effects due to different orbital inclination sizes are smaller than for the seasonal Yarkovsky force and are mostly caused by the increasing variations in $X$ when $I$ is larger.

The rates of $(\mathrm{d} a / \mathrm{d} t)$ and $(\mathrm{d} I / \mathrm{d} t)$ due to the diurnal Yarkovsky force may be very variable. However, for a regolith-covered object with $\rho=1500 \mathrm{~kg} \mathrm{~m}^{-3}$, initial $I=2.5^{\circ}, R=50 \mathrm{~m}$ and a rotation period of $0.5 \mathrm{~h}, a$ may change by typically $(0.5-3) \times$ $10^{-3} \mathrm{AU}$ over a time period of $1 \mathrm{Myr}$. During the same time period, the typical changes in $I$ are approximately $\left(3^{\prime \prime}-15^{\prime \prime}\right)$. The figures for other materials will be lower.

\subsection{Spin vector integration including the Yarkovsky force and non-Yarkovsky force related perturbations}

The dynamical evolution of the orbital elements of a real body is perturbed by several other forces besides the Yarkovsky force. In the case of large asteroids, the most important perturbations are due to gravitational forces.

In order to simulate some of these other effects, an additional perturbation was added to the orbital elements $a$ and $I$. Without the Yarkovsky effect added, $a$ varied periodically between 2.99 AU and 3.01 AU, a full period in 15000 years. Three periodic inclination variations were examined, $0^{\circ}-5^{\circ}$, $7.5^{\circ}-12.5^{\circ}$ and $17.5^{\circ}-22.5^{\circ}$. The initial inclinations were respectively $2.5^{\circ}, 10^{\circ}$ and $20^{\circ}$. In all three cases, the period was $20000 \mathrm{yr}, \Omega$ was still circulating a full $360^{\circ}$ in 25000 years. For middle main belt objects, the main variations in $I$ and $\Omega$ due to gravitational perturbations are typically of these amplitudes and periods, at least when the bodies are unaffected by strong secular or mean motion resonances with the major planets. However, for real bodies, the evolution of $I$ is usually affected significantly by several orbital frequencies. The main period of the variations in $I$ is often the same as the circulating period of $\Omega$. The frequency of the gravitationally related variation in $a$ may be even higher than assumed here. Often, the amplitudes are even smaller than $0.01 \mathrm{AU}$ and varying over time in a very non-uniform way. Real asteroids would of course also experience orbital eccentricity perturbations.

First, the spin vector evolution was integrated including only the induced periodical perturbations and thus, since the Yarkovsky force was excluded, the evolution is independent of object radius. Note though that a spherical shape is still assumed. Regardless of initial $X$, the spin vector evolution is 

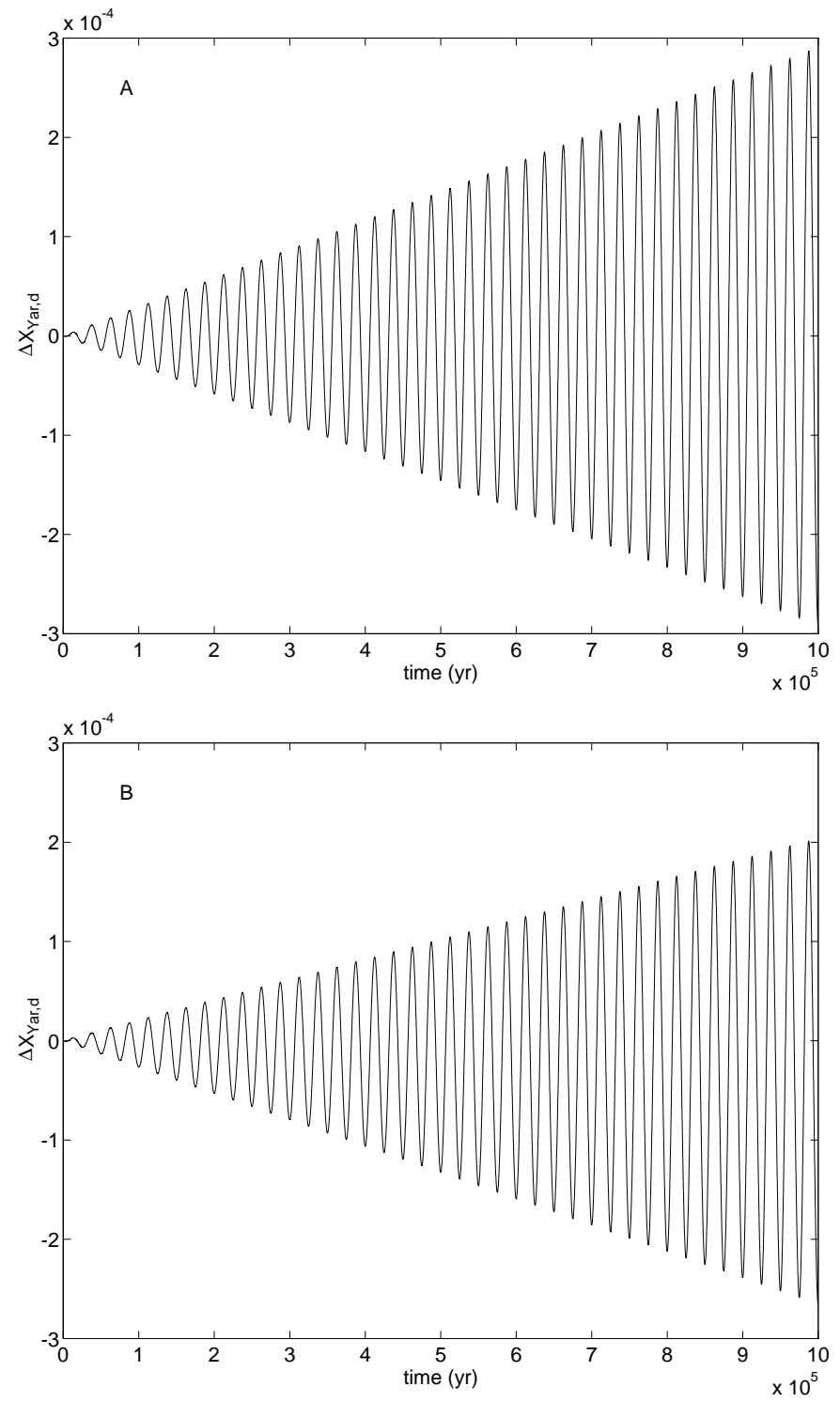

Fig. 3. The differences, $\Delta X_{\text {Yar,d }}$, between the spin vector evolutions excluding and including the diurnal Yarkovsky force $(X=\cos \eta$; where $\eta$ is the obliquity) obtained for a time period of $1 \mathrm{Myr}$ for two hypothetical regolith-covered objects, $\rho=1500 \mathrm{~kg} \mathrm{~m}^{-3}$. Two initial orbital inclinations $(I)$ are used: A: $I=2.5^{\circ}$ and $\mathrm{B}: I=20^{\circ}$. For both objects, the initial $X=0$ and the initial semi-major axis of the orbit $(a)$ is $3 \mathrm{AU}$. The rotation period is $0.5 \mathrm{~h}$. The longitude of the ascending node $(\Omega)$ is circulating a full $360^{\circ}$ in $25000 \mathrm{yr}$. The radius of each object is $50 \mathrm{~m}$. The time-step of the spin-axis integration is $3.125 \mathrm{yr}$. The precession parameter $\alpha=0$. The initial precession angle in longitude $(\psi)$ is 0 .

regular, but not as uniform as when the orbital inclination is constant. The results for a typical object can be seen in Fig. 4. As when the orbital inclination is constant, the variations are larger when the inclination is higher and when initial $X$ is close to 0 .

Then, the orbital and spin vector evolutions were integrated using the same method as before, but combining the changes in the orbital elements due to the Yarkovsky force with those from the periodical perturbations. The differences between the evolution displayed in Fig. 4 and the corresponding one for a $50 \mathrm{~m}$ radius bare basaltic object having the same

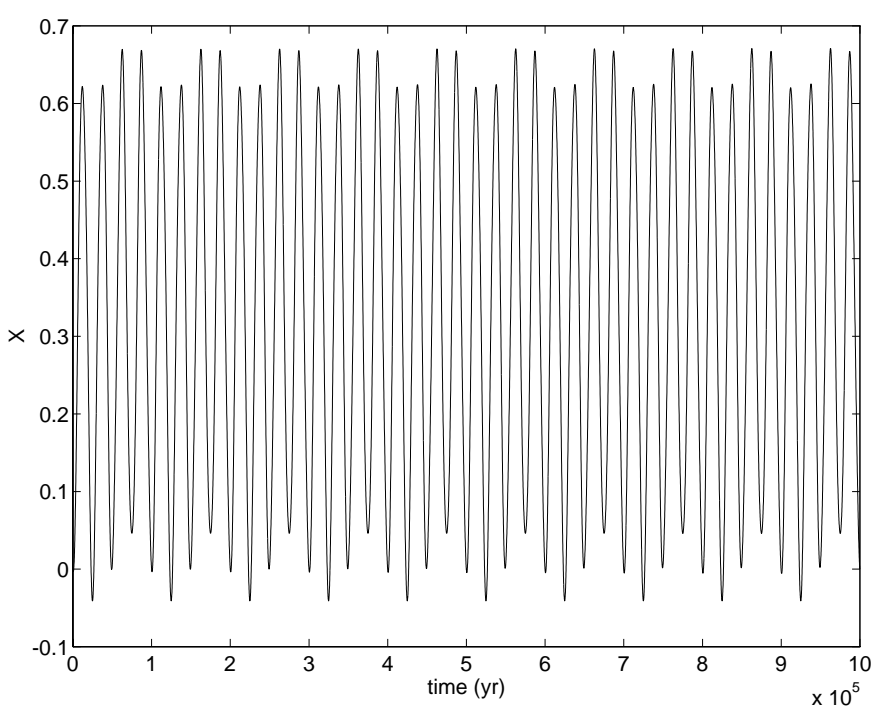

Fig. 4. The $X$-values ( $X=\cos \eta$; where $\eta$ is the obliquity) obtained for a time period of $1 \mathrm{Myr}$ for a hypothetical object. The initial orbital inclination $(I)$ is $20^{\circ}$. The inclination varies in a periodic way between $I=17.5^{\circ}$ and $I=22.5^{\circ}$, the period is $20000 \mathrm{yr}$. The initial semi-major axis of the orbit $(a)$ is $3 \mathrm{AU}$. The semi-major axis of the orbit varies periodically between $a=2.99 \mathrm{AU}$ and $a=3.01 \mathrm{AU}$, the period is $15000 \mathrm{yr}$. The longitude of the ascending node $(\Omega)$ is circulating a full $360^{\circ}$ in $25000 \mathrm{yr}$. The initial $X=0$. The time-step of the spinaxis integration is $3.125 \mathrm{yr}$. The precession parameter $\alpha=0$. The initial precession angle in longitude $(\psi)$ is 0 .

initial conditions but also perturbed by the seasonal Yarkovsky force are presented in Fig. 5. The same tendencies as with the Yarkovsky force as the only perturbing force on $a$ and $I$ can be seen, even though the amplitudes of $\Delta X_{\text {Yar,s }}$ and $\Delta X_{\text {Yar,d }}$ not are changing quite as uniformly with time. However, the magnitude of the differences for a given initial $X$ and initial $I$ is approximately the same as the $\Delta X_{\text {Yar,s }}$ or $\Delta X_{\text {Yar,d }}$ obtained when non-Yarkovsky related perturbations of $a$ and $I$ were omitted, compare Figs. 2A and 5.

\section{Conclusions}

\subsection{Time scales for the spin vector evolution}

Since the differences between models excluding and including the Yarkovsky force tend to increase with time, it is of interest to determine the time likely before the spin direction is changed suddenly in a drastic way due to a collision with another object. Smaller objects can be expected to be much more numerous than larger ones. Thus, during a certain time period an object is more likely to suffer an impact from a small object than from a large one. Naturally, the time expected between two drastic rotational changes is shorter than that of total disruption, which would require much larger impacting objects. These time scales are sensitive to a number of factors related to impacting and spin velocities, material densities and strengths, and sizes and numbers of possible impactors. Most of these factors are highly variable or unknown for many solar system 


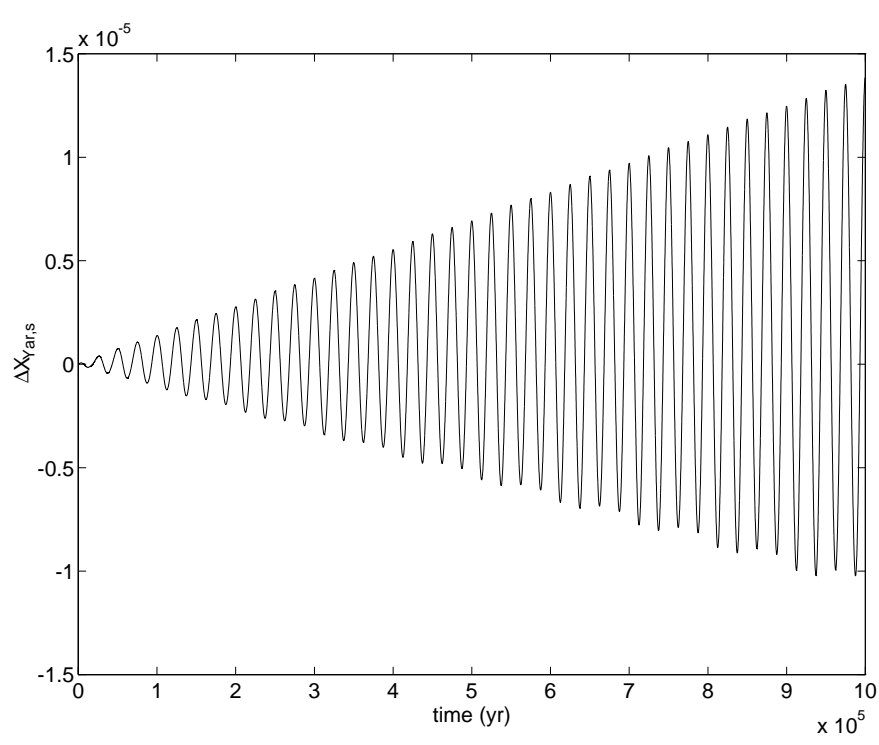

Fig. 5. The differences, $\Delta X_{\text {Yar, },}$, between the spin vector evolutions excluding and including the seasonal Yarkovsky force $(X=\cos \eta$; where $\eta$ is the obliquity) obtained for a time period of 1 Myr for a hypothetical basaltic object. The initial orbital inclination $(I)$ is $20^{\circ}$, the initial semi-major axis of the orbit $(a)$ is $3 \mathrm{AU}$ and the initial $X=0$. The radius of the object is $50 \mathrm{~m}$. The evolution of the orbital elements $a$ and $I$ is also subjected to the induced perturbation not related to the Yarkovsky force also affecting the object in Fig. 4. The longitude of the ascending node $(\Omega)$ is circulating a full $360^{\circ}$ in $25000 \mathrm{yr}$. The time-step of the spin-axis integration is $3.125 \mathrm{yr}$. The precession parameter $\alpha=0$. The initial precession angle in longitude $(\psi)$ is 0 .

regions and bodies. Spin vector changes due to near passages may also be important, especially for planet crossing objects.

Farinella et al. (1998) discussed the time scale, $t_{\text {rot }}$, for totally changing the spin axis of a small main belt object due to collisions. Following this reasoning, first neglect loss of angular momentum carried away by ejecta. Let the density of the impacting object be the same as that of the target. Now, with a size-independent rotational period of 5 h, Farinella et al. (1998) estimated this time scale as:

$t_{\text {rot }}=R^{4 / 3}\left(1.88 \times 10^{4}\right)$

with the results given in years.

For the objects in this study, supposing an impactor density of $3500 \mathrm{~kg} \mathrm{~m}^{-3}, t_{\text {rot }} \approx 3 \mathrm{Myr}$ for a basaltic body with a $50 \mathrm{~m}$ radius, $9 \mathrm{Myr}$ for a $100 \mathrm{~m}$ radius object and $0.2 \mathrm{Gyr}$ for an $1 \mathrm{~km}$ radius object. However, for the smaller objects, it may instead be more realistic to adopt a spin period that depends on the object radius (see Farinella et al. 1998). At least some small objects may be very fast rotators (Ostro et al. 1999; Pravec et al. 2000). Assuming a connection between spin rate and radius, $\omega \propto R^{-1}$, and a spin period of $5 \mathrm{~h}$ when $R=0.5 \mathrm{~km}$, which implies a spin period of $0.5 \mathrm{~h}$ for a $50 \mathrm{~m}$ radius object, Farinella et al. (1998) now estimated $t_{\text {rot }}$ as:

$t_{\text {rot }}=R^{1 / 2}\left(3.34 \times 10^{6}\right)$

still with the results given in years.

Now $t_{\text {rot }} \approx 24 \mathrm{Myr}$ for a basaltic $50 \mathrm{~m}$ radius object and 33 Myr for a $100 \mathrm{~m}$ radius object. For iron-rich bodies with their heigher densities $\left(\sim 8000 \mathrm{~kg} \mathrm{~m}^{-3}\right)$, still assuming a projectile density of $3500 \mathrm{~kg} \mathrm{~m}^{-3}$, all these time scales should be increased by a factor $\sim 2$. Analogously, a lower target density of $1500 \mathrm{~kg} \mathrm{~m}^{-3}$ would decrease the time scales by a factor $\sim 2$. Since the effects from the seasonal Yarkovsky force on bare basaltic objects are approximately 2-3 times larger than on iron-rich ones during the same time period, this force may be of about the same importance for the spin vector evolution of both classes of bodies. The diurnal effects would be most important for regolith-covered objects, while the effects on bare ones would be smaller. Note that a substantial loss of angular momentum at the impact or a lower projectile density would increase the time scales above to some extent. Also, remember that the time of dynamical relaxation may be of the same order as $t_{\text {rot }}$ for many real subkilometer sized asteroids.

\subsection{Discussion}

After $30 \mathrm{Myr}$, the maximum $\Delta X_{\mathrm{Yar}, \mathrm{s}}$-value for a bare basaltic object with $R=50 \mathrm{~m}$ will be $\sim\left(10^{-5}-10^{-4}\right)$ for an initial $I=2.5^{\circ}$ and $\sim\left(10^{-4}-5 \times 10^{-4}\right)$ for an initial $I=20^{\circ}$, depending on initial $X$. For an equal sized regolith-covered object, with $\rho=1500 \mathrm{~kg} \mathrm{~m}^{-3}$ and a rotation period of $0.5 \mathrm{~h}$, the maximum $\Delta X_{\text {Yar, }}$ after the same time is $\sim\left(10^{-3}-10^{-2}\right)$. When the bulk density is higher, $\Delta X_{\text {Yar,d }}$ is still smaller. The non-Yarkovsky related variations in $X$ are typically $\sim(10-100)$ times larger (Fig. 1). No numerical integrations longer than $30 \mathrm{Myr}$ were performed. However, the amplitudes of $\Delta X_{\text {Yar, } \mathrm{s}}$ and $\Delta X_{\text {Yar,d }}$ seem to increase approximately in a linear way with time, even for orbital evolutions affected by the induced non-Yarkovsky force related perturbations (Fig. 5). Thus, it may be possible to estimate these amplitude variations over a longer time period, at least for larger bodies with small Yarkovsky effect related perturbations. Increasing the radius to $1 \mathrm{~km}$ may, for the bare basaltic objects, cause a maximum deviation in $X$ due to the seasonal Yarkovsky force of $\sim\left(10^{-6}-10^{-4}\right)$ after $100 \mathrm{Myr}$. A $1500 \mathrm{~kg} \mathrm{~m}^{-3}$ regolith- covered object of this size, with a rotation period of $10 \mathrm{~h}$, may have a maximum $\Delta X_{\text {Yar,d }}$ of about $\sim\left(10^{-4}-2 \times 10^{-3}\right)$ after the same time. If the radius is increased to $10 \mathrm{~km}$, the maximum $\Delta X_{\text {Yar, }}$ obtained after the present age of the solar system $(4.5 \mathrm{Gyr})$ may be estimated to $\sim\left(10^{-3}-10^{-2}\right)$.

In this study, $\Omega$ is assumed to be circulating with a period of $25000 \mathrm{yr}$. Naturally, the real objects will all have their own orbital rates of change. The typical period seems to be (20 000-30 000) yr for most asteroids at this distance ( 3 AU) from the Sun. It was found that the maximum size of the deviations in $X$ over a longer time period was insensitive to the size of $(\mathrm{d} \Omega / \mathrm{d} t)$. The increasing differences between the orbital inclination evolutions over time can be expected to be the main reason for the divergences between the models.

The effects from the Yarkovsky force on the size of the obliquity variations seem to be negligible, at least for kilometer sized and larger asteroids, i.e. for objects whose spin properties have been measured and used in e.g. statistical studies. For smaller objects, Yarkovsky forces may affect the spin vectors to a larger extent, but since $t_{\text {rot }}$ is shorter for such bodies, 
the influences on the spin vector from other forces seem to be dominating. Also, very little is known of the spin properties of real 50-100 meter sized bodies in the solar system.

The linearized model will slightly overestimate the effects from the seasonal Yarkovsky force (Rubincam 1995). According to the nonlinearized theory by Vokrouhlický \& Farinella (1998b), this overestimation is $\sim 15 \%$ for thermally "large" bodies. A higher albedo than used here $(A=0)$ would also diminish the effects from the Yarkovsky force. The effects on the spin vector evolution of real asteroids may thus be even smaller than found here. It is therefore concluded that in most cases a model including the gravitational perturbations from the major planets but excluding the Yarkovsky force is sufficient when the dynamical spin vector evolution of objects like those in this study is examined. Naturally, a complete evolutional model must include both dynamical and collisional factors, and for certain classes of objects also the YORP effect and/or tidal forces due to near passages.

The investigations have been restricted to spherical bodies. In addition, circular orbits have been assumed throughout the integrations. However, the investigated spin vector evolution is not directly dependent on the orbital eccentricity when spherical bodies are examined (Eq. (37)). In fact, the influences from the eccentricity on the spin vector evolution are always small unless the eccentricity is high. Note though that a higher eccentricity would influence the Yarkovsky related evolution of the orbital elements to some extent (e.g. Spitale \& Greenberg 2001).

The semi-major axis of the orbit is also affecting the results. In this study, the concentration have been on objects where initial $a=3$ AU. For objects closer to the Sun, the importance of the Yarkovsky force will be increased. Repeating the numerical integrations for objects with initial $a=2$ AU showed that the appearance of $\Delta X_{\text {Yar,d }}$ and $\Delta X_{\text {Yar,s }}$ were qualitatively similar but larger by, depending on the physical, thermal and orbital parameters, a factor $\sim(1-3.5)$.

The differences between a spherical and a spheroidal body model were examined by Vokrouhlický \& Farinella (1998b) regarding the seasonal effect. Assuming objects of the same sizes and moderate flattenings, the rate of $(\mathrm{d} a / \mathrm{d} t)$ could be up to a factor 2 larger or smaller for a spheroidal object as compared to a perfect sphere. Even for quite extreme flattenings, this factor was only between 2 and 3. Vokrouhlický (1998b) found similar results for the diurnal Yarkovsky force. For large main belt objects, the precession parameter $\alpha$ is typically $(10-20)^{\prime \prime} \mathrm{yr}^{-1}$, although both higher and lower values may be found (Skoglöv et al. 1996). Since $\alpha \propto a^{-3}$, the Yarkovsky effects on the semimajor axis of the orbit may influence $\alpha$ and thus the precession frequency $(\mathrm{d} \psi / \mathrm{d} t)$ to a higher extent for certain non-spherical bodies. However, this will mainly affect the frequency of the $X$-oscillations and not so much the size of the amplitudes.

The comparatively small differences between a spherical and a non-spherical model makes it reasonable to assume that the spherical model gives a good representation of the effects of the Yarkovsky force on the spin vector evolution of spheroidal main belt bodies, especially when regarding the amplitudes of the $X$-oscillations.
It is not known whether or not a regolith layer is normally present on (50-1000) $\mathrm{m}$ sized bodies, neither is the normal size of this layer if present. It is reasonable to suspect that smaller bodies are less likely to be regolith covered than larger ones, due to lower gravities and likely faster rotations. The observations of and models used for real objects, usually larger than those considered in this study, seem to indicate a large variation in conditions (e.g. McFadden et al. 1989). The thermal skin depth, $l_{\mathrm{S}}$, i.e. the characteristic distance of solar radiation penetration is (regarding the seasonal effect):

$l_{\mathrm{S}}=K^{1 / 2} /(\rho n C)^{1 / 2}$.

For main belt bodies (seasonal effect), this depth is a couple of meters for a bare basaltic object and $\sim(10-20) \mathrm{m}$ for an ironrich one. For a regolith covered object, it is $\sim(0.1-0.2) \mathrm{m}$. Thus, a body with a regolith cover smaller than about this size may be considered "bare" when the seasonal Yarkovsky force is considered. Note though that already a regolith cover of a few centimeters could disturb the thermal wave.

A faster rotation of the (50-100) m radius objects which would increase $t_{\text {rot }}$ also seems to diminish the probability of a regolith layer and thus increase the importance of the seasonal effect as opposed to the diurnal.

It seems that the dynamical spin axis evolution of the main belt asteroids generally is regular (Skoglöv et al. 1996; Skoglöv $\&$ Erikson 2002). However, this is not the case for the inner solar system asteroids (Skoglöv 1997, 1999), where the spin vector evolution often is chaotic and very sensitive to the initial parameters used. Thus, a small disturbance, e.g. due to Yarkovsky forces, could influence the spin vector evolution of such objects in a non-negligible way. However, the expected lifetime of these objects is short, and the orbital evolutions are in general also subjected to chaos. It seems therefore probable that the Yarkovsky force related influences on the sizes and locations of the chaos influenced zones in initial $X$ will be small or negligible. Note also that for planet-crossing asteroids with very high orbital eccentricities, the spin axis evolution often seems to be subjected to chaos and experience large and fast changes regardless of initial spin vector direction due to purely gravitational forces (Skoglöv 1999).

Acknowledgements. I want to thank Joseph Spitale for a valuable and helpful review.

\section{References}

Bottke, Jr, W. F., Rubincam, D. P., \& Burns, J. A. 2000, Icarus, 145, 301

Burns, J. A., \& Safronov, V. S. 1973, MNRAS, 165, 403

Burns, J. A., Lamy, P. L., \& Soter, S. 1979, Icarus, 40, 1

Farinella, P., Vokrouhlický, D., \& Hartmann, W. K. 1998, Icarus, 132, 378

Harris, A. 1994, Icarus, 107, 209

Laskar, J., \& Robutel, P. 1993, Nature, 361, 608

Laskar, J., Joutel, F., \& Robutel, P. 1993, Nature, 361, 615

McFadden, L.-A., Tholen, D., \& Veeder, G. 1989, in Asteroids II, ed. R. P. Binzel, T. Gehrels, \& M. S. Matthews (University of Arizona Press, Tucson), 442 
Öpik, E. J. 1951, Proc. Roy. Irish Acad., 54, 165

Ostro, S. J., Pravec, P., Benner, L. A. M., et al. 1999, Science, 285, 557

Pravec, P., Hergenrother, C., Whiteley, R., et al. 2000, Icarus, 147, 477

Rabinowitz, D. L., Gehrels, T., Scotti, J. V., et al. 1993, Nature, 363, 704

Rubincam, D. P. 1995, J. Geophys. Res., 100, 1585

Rubincam, D. P. 1998, J. Geophys. Res., 103, 1725

Rubincam, D. P. 2000, Icarus, 148, 2

Skoglöv, E. 1997, Planet. Space Sci., 45, 439

Skoglöv, E. 1999, Planet. Space Sci., 47, 11
Skoglöv, E., \& Erikson, A. 2002, Icarus, in press

Skoglöv, E., Magnusson, P., \& Dahlgren, M. 1996, Planet. Space Sci., 44, 1177

Spitale, J., \& Greenberg, R. 2001, Icarus, 149, 222

Spitale, J., \& Greenberg, R. 2002, Icarus, 156, 211

Vokrouhlický, D. 1998a, A\&A, 335, 1093

Vokrouhlický, D. 1998b, A\&A, 338, 353

Vokrouhlický, D. 1999, A\&A, 344, 362

Vokrouhlický, D., \& Broz, M. 1999, A\&A, 350, 1079

Vokrouhlický, D., \& Farinella, P. 1998a, A\&A, 335, 351

Vokrouhlický, D., \& Farinella, P. 1998b, A\&A, 116, 2032 